\title{
Maintenance of inspiratory muscle training in COPD patients: one year follow-up
}

\author{
P. Weiner, R. Magadle, M. Beckerman, M. Weiner, N. Berar-Yanay
}

Maintenance of inspiratory muscle training in COPD patients: one year follow-up. P. Weiner, R. Magadle, M. Beckerman, M. Weiner, N. Berar-Yanay. C) ERS Journals Ltd 2004.

ABSTRACT: In most chronic obstructive pulmonary disease (COPD) patients, dyspnoea and functional exercise capacity may improve as a result of inspiratory muscle training (IMT). However, the long-term benefits of IMT have been investigated to a much lesser extent.

The present study investigated the short-term and long-term benefits of IMT on inspiratory muscle performance (strength and endurance), exercise capacity and the perception of dyspnoea.

Thirty-eight patients with significant COPD had 3 months of basic IMT and were then randomised into a group that received maintenance IMT for the next year, and a group that got training with very low load. Following the basic training there was a statistically significant increase in inspiratory muscle performance, 6-min walk test (6MWT), and a decrease in the dyspnoea. During the second stage of the study, the training group continued to maintain the improvement in all parameters, while there was already deterioration in the inspiratory muscle performance, exercise capacity and dyspnoea in the low intensity group during the 6-12 month period.

The present study concludes that, in patients with significant chronic obstructive pulmonary disease, inspiratory muscle training results in improvement in performance, exercise capacity and in the sensation of dyspnoea. The benefits of 12-weeks of inspiratory muscle training decline gradually over 1 yr of follow-up if maintenance training is not performed.

Eur Respir J 2004; 23: 61-65.
Dept of Medicine A, Hillel Yaffe Medical Center, Hadera, Israel.

Correspondence: P. Weiner

Dept of Medicine A

Hillel Yaffe Medical Center

Hadera

Israel 38100

Fax: 01197246304522

E-mail: weiner@hillel-yaffe.health.gov.il

Keywords: Inspiratory muscle training sensation of dyspnoea exercise performance

Received: May 292003

Accepted after revision: September 12003
Patients with significant chronic obstructive pulmonary disease (COPD) have respiratory and peripheral muscle weakness [1]. Respiratory muscle weakness may contribute to dyspnoea and to poor exercise performance [2, 3]. Therefore, it was rational to try ventilatory muscle training in these patients, to enhance respiratory muscle function and potentially reduce the severity of breathlessness and improve exercise tolerance.

Inspiratory muscle training has been extensively investigated in patients with COPD. Although, in their meta-analysis, SMITH et al. [4] concluded that inspiratory muscle training (IMT) in patients with COPD did not reveal significant treatment effect, it was later shown, in studies in which the training stimulus was adequate, that in most COPD patients, dyspnoea and functional exercise capacity may improve as a result of such training [5-7]. The joint American College of Chest Physicians/American Association of Cardiovascular and Pulmonary Rehabilitation committee [8] declared that when the stimulus or load placed on the respiratory muscles during training is sufficient to augment inspiratory muscle strength, there is an associated increase in exercise capacity and decrease in dyspnoea. In a recent meta-analysis performed by LÖTTERS et al. [9] it was concluded that IMT, alone or as an adjunct to general exercise reconditioning, decreases dyspnoea and improves functional exercise capacity in patients with COPD.

For editorial comments see page 5.
Despite the documented benefits of IMT in a rehabilitation program, longer-term effects are less clearly defined. While it is well documented that the benefits of multidisciplinary pulmonary rehabilitation gradually decline over 1 or 2 yrs [10-11], the long-term effects of IMT were investigated to a much lesser extent in COPD.

The present study was performed in order to investigate the short-term and the long-term effect of IMT on inspiratory muscle performance, exercise performance and the perception of dyspnoea, and the decline of these parameters following withdrawal from the maintenance training program in patients with significant COPD.

\section{Patients and methods}

\section{Subjects}

Thirty-eight consecutive patients, 31 males and 7 females, with spirometric evidence of significant chronic air-flow limitation (forced expiratory volume in one second (FEV1) $<50 \%$ predicted, FEV1/forced vital capacity (FVC) $<70 \%$ pred) who were diagnosed as having COPD according to the criteria of the American Thoracic Society [12], were recruited from the community for the study. The eligibility rate was $65 \%$. According to the statistician's power calculation a group size of 30 patients would allow the observation of significant differences between the groups. 
They were all new to a rehabilitation program, and none had any additional regular exercise or dietary supplements. All were on regular long-acting bronchodilators, and 28 were on regular inhaled corticosteroid therapy.

They were all observed during a 4-week run-in period, when their regular treatment was maintained, to verify stability in their clinical and functional status. Their characteristics are summarised in table 1. Patients with cardiac disease, poor compliance, a requirement of supplemental oxygen, or carbon dioxide retention (carbon dioxide arterial tension $>6.65 \mathrm{kPa}$ $(>50 \mathrm{mmHg})$ ) were excluded from the study.

\section{Study design}

All tests were performed before and 3, 6, 9, 12, and 15 months after starting the training period.

After completion of 3 months of IMT the patients were randomised, using a random numbers table, into two groups: a group of patients assigned to receive IMT for the next year, and a group of patients assigned as a control group, who got training with very low load.

In all the patients several practice tests were performed before the baseline value in order to correct possible training and learning effect. All the data was collected by the same collector, who was blinded to the training group, as well as by the patients themselves, who were also blinded to the mode of treatment.

The study protocol was approved by the institutional ethics committee and informed consent was obtained from all the subjects.

\section{Tests}

Spirometry. The FVC and the FEV1 were measured 3 times on a computerised spirometer (Compact, Vitalograph, Buckingham, England) and the best trial is reported.

6-minute walk test. The distance the patient was able to walk in 6 min was determined in a measured corridor as described for the 12-min walk test by MCGAVIN et al. [13]. The patients were instructed to walk at their fastest pace and cover the longest possible distance over $6 \mathrm{~min}$ under the supervision of a physiotherapist. The test was performed twice and the best result is reported.

Table 1.-Characteristics of patients with chronic obstructive pulmonary disease

\begin{tabular}{lcc}
\hline & IMT & Control \\
\hline Subjects n & 19 & 19 \\
Age yr & $66.3 \pm 3.4$ & $64.9 \pm 3.2$ \\
Sex M:F & $15: 4$ & $16: 3$ \\
Weight kg & $74.2 \pm 2.9$ & $73.3 \pm 3.6$ \\
Height m & $1.69 \pm 3.4$ & $1.67 \pm 3.1$ \\
FVC L & $2.37 \pm 0.8$ & $2.44 \pm 0.7$ \\
FVC L \% pred & $66 \pm 4.2$ & $71 \pm 4.0$ \\
FEV1 L & $1.31 \pm 0.3$ & $1.39 \pm 0.4$ \\
FEV1 L \% pred & $45 \pm 2.6$ & $47 \pm 2.2$ \\
6MWT m & $262 \pm 44$ & $274 \pm 45$ \\
PI,max cmH & $66 \pm 4.7$ & $63 \pm 4.0$ \\
Current smokers & 6 & 4 \\
Exsmokers & 12 & 13 \\
\hline
\end{tabular}

Values are expressed as mean \pm SEM. IMT: inspiratory muscle training; M: male; F: female; FVC: forced vital capacity; FEV1: forced expiratory volume in one second; 6MWT: 6-min walk test; PI,max: maximal inspiratory mouth pressure; pred: predicted.
Inspiratory muscle strength. Inspiratory muscle strength was assessed by measuring the maximal inspiratory mouth pressure $(P I, \max )$ at residual volume, as previously described by BLACK and HYATT [14]. Mouth pressures were measured with a vacuumed 1002 mouthpiece (Vista, Ventura, CA, USA), that has a small air leak to prevent pressure generation by glottis closure, connected to a pressure transducer (1050 BP transducer; Biopac Systems Inc, Santa Barbara, CA, USA) and recorded on a strip chart recorder. The value obtained from the best of at least three efforts was used.

Inspiratory muscle endurance. To determine inspiratory muscle endurance a device similar to that proposed by NICKERSON and KEENS [15] was used. Subjects inspired through a two-way Hans-Rudolph valve (Hans-Rudolph, Kansas City, MO, USA) whose inspiratory port was connected to a chamber and plunger to which weights could be added externally. Inspiratory elastic work was then increased by the progressive addition of 25-100 g weights (to get 5-7 measurements during the test), at 2-min intervals, as previously described by MARTYN et al. [16], until the subjects were exhausted and could no longer inspire. The pressure achieved with the heaviest load (tolerated for at least $60 \mathrm{~s}$ ) was defined as the peak inspiratory pressure (PIP).

Dyspnoea. Dyspnoea was assessed using two techniques: 1) dyspnoea in daily activities was assessed with Mahler's baseline dyspnoea index (BDI) [17], and by the transition dyspnoea index (TDI), following training. 2) the perception of dyspnoea (POD) was also measured while the subject breathed through the same device proposed by NICKERSON and KEENS [15]. The subjects breathed against progressive resistance, at 1 min intervals, in order to achieve mouth pressure of 0 (no resistance), 5 , 10, 20 and $30 \mathrm{cmH}_{2} \mathrm{O}$. After breathing for 1 min in each inspiratory load, in a protocol similar to the one previously described by the current authors' group [18], the subjects rated the sensation of difficulty in breathing (dyspnoea) using a modified Borg scale [19]. This is a linear scale of numbers ranking the magnitude of difficulty in breathing, ranging from 0 (none) to 10 (maximal).

\section{Training protocol}

Stage I. All subjects trained daily, six times a week, each session consisting of $30 \mathrm{~min}$, for 3 months. The training was performed using a threshold inspiratory muscle trainer (Threshold $^{\mathrm{TM}}$ Inspiratory Muscle Trainer, Healthscan, New Jersey, NJ, USA). They started breathing at a resistance that required generation of $15 \%$ of their $P \mathrm{I}$,max for 1 week. The load was then increased incrementally, $5-10 \%$ each session, to reach generation of $60 \%$ of their $P \mathrm{I}$,max at the end of the first month. Specific IMT (SIMT) was then continued at $60 \%$ of their $P \mathrm{I}$, max adjusted weekly to the new $P \mathrm{I}$,max achieved.

The training was performed in the current authors' rehabilitation centre for 1 month under the supervision of a respiratory therapist followed by home training, verified by a respiratory therapist, daily by phone, and once weekly by a personal visit, for the next 2 months.

Stage II. At the end of 3 months IMT, the patients were randomised into an IMT group and a control group that got training with very low load.

The IMT group continued to train three times a week, each session consisting of $30 \mathrm{~min}$, for the next 12 months at $60 \%$ of their PI,max, adjusted monthly to the new PI,max achieved.

The control group trained for the same sessions with a fixed load that required generation of mouth pressure of $7 \mathrm{cmH}_{2} \mathrm{O}$.

The training was performed at home, verified by the 
respiratory therapist, daily by phone, and once weekly by a personal visit, for the next year.

\section{Data analysis}

The results are expressed as mean \pm SEM. Comparisons of lung function, respiratory muscle strength and endurance, the 6-min walk test (6MWT), and rating of dyspnoea, within and between the two groups were carried out using the two-way repeated measures analysis of variance.

\section{Results}

There were no significant differences between the two groups in age, height, weight, mean baseline FEV1 and FVC, PI,max, or PIP, and the 6MWT, at the beginning of the study. Six patients dropped out of the study during the basic training period (one of whom died and three had exacerbations) so the results reported at the end of the first stage are on the 32 remaining patients. The attendance rate in the first stage was $83 \% \pm 7 \%$. During the next year four patients from the training group dropped out of the study (two of whom died) and seven patients from the control group (four of whom died). The attendance rate in the second stage of the study was $72 \% \pm 7 \%$ in the training group and $69 \% \pm 8 \%$ in the control group.

\section{Spirometry}

Following the basic training period, there was no significant change in the FEV1 or in the FVC either in the training groups or in the control group. Following the second stage of the study there was a statistically insignificant trend for decrease in FEV1 and FVC in the control group but not in the study group (fig. 1). There was a statistically significant difference between the groups at the ninth months stage.

\section{Inspiratory muscle strength and endurance}

Following the basic training period there was a similar statistically significant increase in $P \mathrm{I}$, max in the training and the control groups (from $66 \pm 4.7 \mathrm{cmH}_{2} \mathrm{O}$ to $87 \pm 5.3 \mathrm{cmH}_{2} \mathrm{O}$ and from $63 \pm 4.0 \mathrm{cmH}_{2} \mathrm{O}$ to $84 \pm 4.9 \mathrm{cmH}_{2} \mathrm{O}$, respectively $\mathrm{p}<0.005$ ) with no difference between the groups. During the

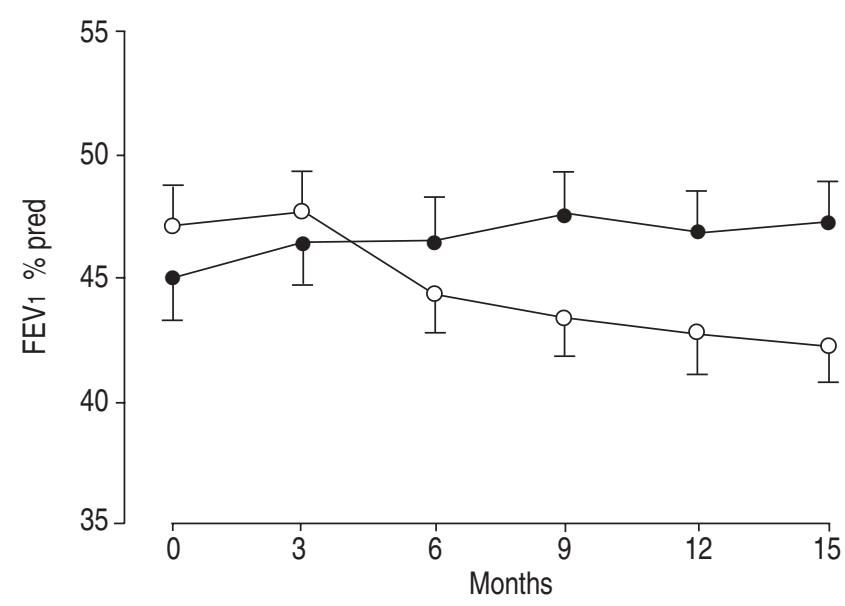

Fig. 1. - Mean \pm SEM forced expiratory volume in one second (FEV1) before and following the training period (basic training between 0 and 3 months). - training group; $\bigcirc$ : control group. second stage of the study the training group continued to show a small increase in the PI,max, while already at the 6 month period there was a significant decrease in the $P \mathrm{I}, \max$ in the control group $(\mathrm{p}<0.01)$ with a significant difference between the groups $(p<0.01)$. This difference was maintained until the end of the fifteenth month (fig. 2).

The inspiratory muscle endurance, as assessed by the PIP showed the same pattern. While following the basic training period there was a similar statistically significant increase in PIP in the training and the control groups, during the second stage of the study the training group continued to show a small increase in the PIP, while the control group showed a continuous decrease in the PIP (fig. 3).

\section{6-minute walk test}

There was statistically significant improvement in the 6MWT following the basic training period, in both groups.

During the second stage of the study there was a gradual

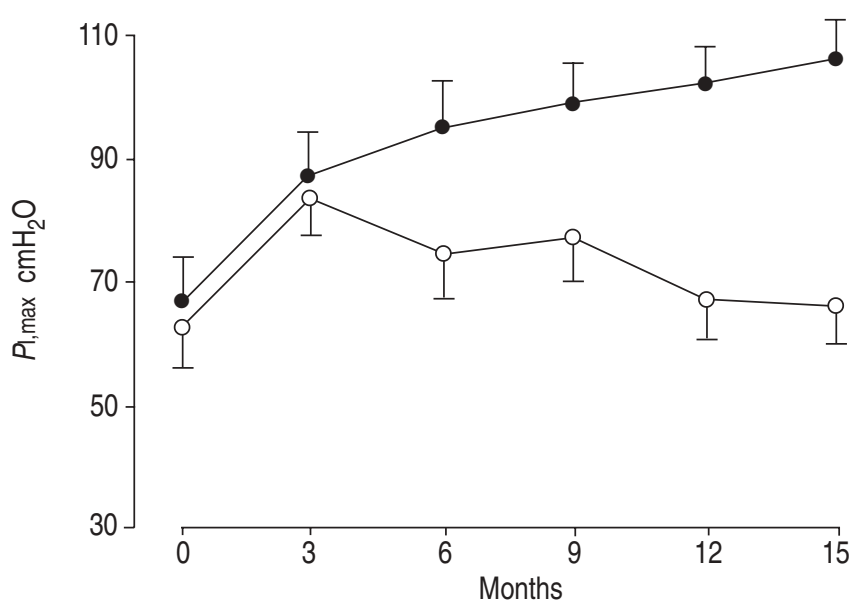

Fig. 2. - Inspiratory muscle strength, as assessed by the maximal mouth inspiratory pressure $\left(P_{\mathrm{I}}, \mathrm{max}\right)$, before and following the training period (basic training between 0 and 3 months). Already at the 6 month period there was a significant difference between the groups $(\mathrm{p}<0.01)$. : training group; $\bigcirc$ : control group.

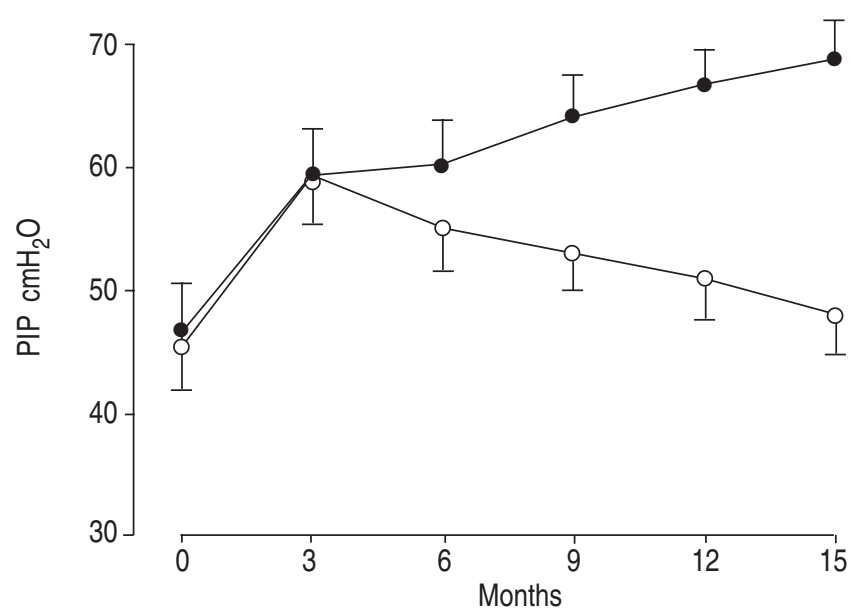

Fig. 3. - Mean \pm SEM inspiratory muscle endurance, as assessed by the peak inspiratory pressure (PIP) (pressure during the highest load), before and following the training period (basic training between 0 and 3 months). Already at the 6 month period there was a significant difference between the groups $(\mathrm{p}<0.01)$. 0 : training group; $\bigcirc$ : control group. 


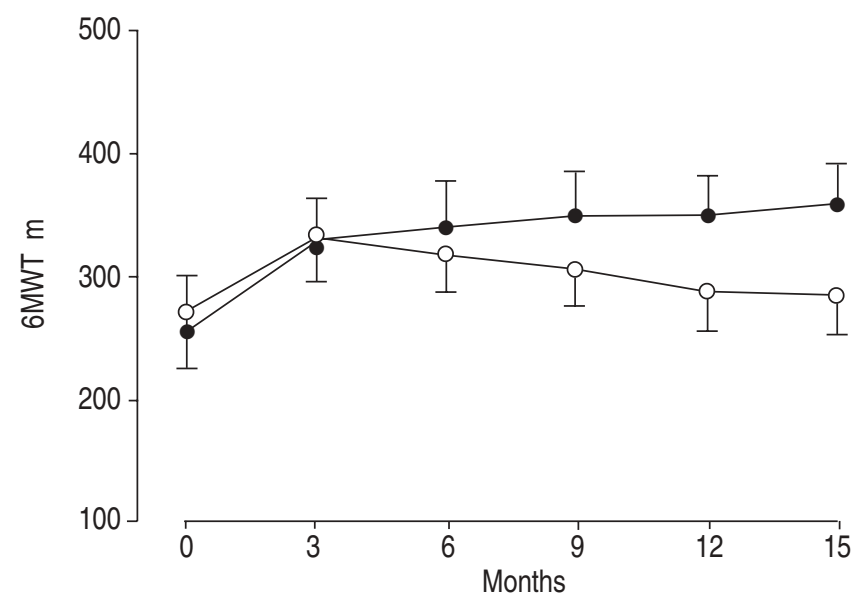

Fig. 4. - The Mean \pm SEM distance walked in 6 min (6MWT) before and following the training period (basic training between 0 and 3 months). The difference between the groups became statistically significant at the end of the twelfth month. 0 : training group; $O$ : control group.

decrease in the 6MWT in the control group but not in the training group. The difference between the groups became statistically significant at the end of the twelfth month (fig. 4).

\section{Dyspnoea}

Mahler's baseline dyspnoea index. There was no statistically significant difference between the two groups in the different components of the BDI.

Following the basic training period there was a similar statistically significant increase in the TDI in the training and the control groups. During the second stage of the study the training group continued to show the same degree of TDI, while the control group returned to the baseline values (table 2).

Perception of dyspnoea. There was no difference in the POD between the two groups before training. A statistically significant decrease in the mean Borg score during breathing against resistance was associated with the increase in the inspiratory muscle strength, following the basic training.

During the second stage of the study the training group continued to show a similar degree of Borg score during breathing against resistance increase while at the 9 month period there was already a significant increase in the POD in the control group $(\mathrm{p}<0.01)$ with a significant difference between the groups $(\mathrm{p}<0.01)$. This difference was maintained until the end of the fifteenth month (fig. 5).

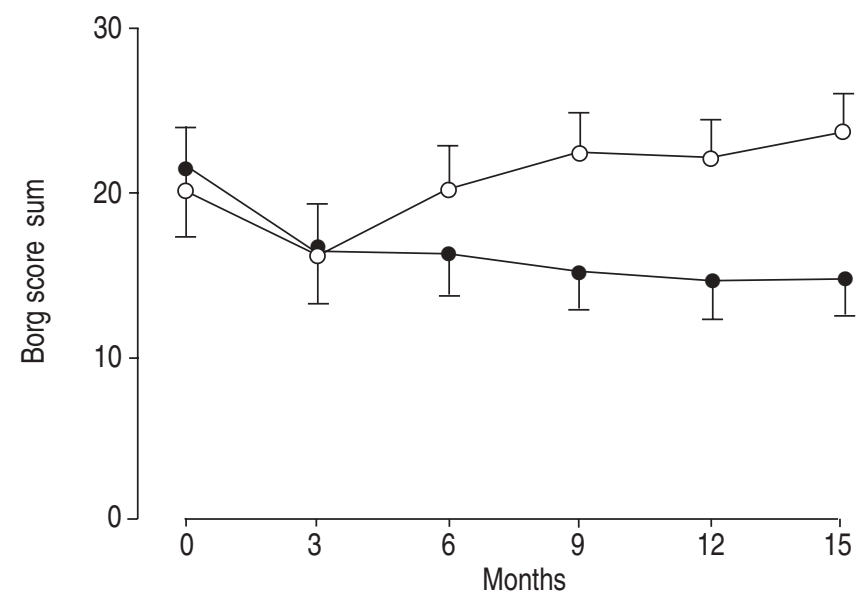

Fig. 5. - Baseline mean \pm SEM perception of dyspnoea (Borg score) during breathing against load in all chronic obstructive pulmonary disease patients, and following training in the two groups (basic training between 0 and 3 months). The difference between the groups became statistically significant at the end of the ninth month. training group; $\bigcirc$ : control group.

\section{Discussion}

This study shows that in patients with significant COPD, inspiratory muscles can be trained with improvement of both muscle strength and endurance. The improvement in the inspiratory muscle performance is associated with improved exercise performance and in the sensation of dyspnoea in daily activities with no improvement in pulmonary function. The benefits of a 12-week inspiratory muscle training decline gradually over 1 year of follow-up when maintenance training is not performed.

All of the patients had at least some degree of inspiratory muscle weakness. Inspiratory muscle weakness is found in the majority of patients with significant COPD [2]. Patients with COPD must breathe at high lung volumes to maintain patency of their narrowed airways. The major abnormality of respiratory muscle function in these patients is thought to be the mechanical disadvantage caused by this hyperinflation. Hyperinflation depresses the dome of the diaphragm, shortens its fibres, and forces it to work on an ineffective portion of its length/tension curve.

The results of the basic training program parallel other studies in which significantly increased inspiratory muscle performance was associated with improved exercise tolerance and decreased dyspnoea [9]. The improvements observed in the present study are mainly physiological and the translation of those benefits to daily activities are yet to be investigated.

Most of the benefits of training in the present study did not persist 12 months after the basic training if maintenance training was not applied. PI,max, PIP, 6MWT and the POD

Table 2.-Baseline and following training values for dyspnoea in the training groups and in the control group

\begin{tabular}{|c|c|c|c|c|c|c|}
\hline & \multicolumn{3}{|c|}{ IMT } & \multicolumn{3}{|c|}{ Control } \\
\hline & BDI & TDI (3rd) & TDI (15th) & BDI & TDI (3rd) & TDI (15th) \\
\hline Functional impairment & $1.9 \pm 0.2$ & $0.4 \pm 0.1$ & $0.4 \pm 0.1$ & $1.7 \pm 0.2$ & $0.6 \pm 0.1$ & $0.2 \pm 0.1$ \\
\hline Magnitude of task & $1.8 \pm 0.2$ & $0.7 \pm 0.1$ & $0.8 \pm 0.1$ & $1.7 \pm 0.3$ & $0.6 \pm 0.1$ & $-0.1 \pm 0.1$ \\
\hline Magnitude of effort & $1.8 \pm 0.2$ & $0.5 \pm 0.1$ & $0.5 \pm 0.1$ & $1.8 \pm 0.2$ & $0.6 \pm 0.1$ & $0.2 \pm 0.1$ \\
\hline Focal score & $5.5 \pm 0.7$ & $1.6 \pm 0.2$ & $1.7^{\#} \pm 0.2$ & $5.2 \pm 0.8$ & $1.8 \pm 0.2$ & $0.3 \pm 0.1$ \\
\hline
\end{tabular}

Values are expressed as mean \pm SEM. $n=12$ for inspiratory muscle training (IMT) group and $n=9$ for the control group. Baseline dyspnoea index (BDI) before training; transition dyspnoea index (TDI) 3rd, 15th following training. \#: Statistically significant difference between the groups. 
decreased gradually and this decrease became statistically significant 9 months, 12 months, 12 months and 6 months following the completion of the basic training, respectively.

The outcomes of the current study could have been influenced by learning effects due to the type of training. However, in all the patients several practice tests were performed before the baseline value in order to correct possible training and learning effect. Also, it can be expected that if a learning effect plays a role in the outcomes it would be the same in the various groups of patients.

Several studies have evaluated longer-term general rehabilitation interventions, with mixed results. However, IMT was included in only some of them. In the study of GUELL et al. [20] the benefits of pulmonary rehabilitation were present but diminished over the second year of follow-up. TROOSTERS et al. [10] have shown that 6 months of general training was associated with improved exercise tolerance and quality of life that persisted over the next year. Two other studies [21, 22] were unable to show long-term benefits of general pulmonary rehabilitation.

Failure to obtain long-term benefits from short-term intervention is not limited to pulmonary or exercise training. Long-term maintenance of behavioural changes have also been difficult to demonstrate in research on smoking cessation [23], weight loss [24], or exercise adherence [25].

In the current study, the improvement in the dyspnoea indexes and in functional exercise capacity, following the basic training, was not only statistically significant but also exceeded the minimal clinically-important difference for improvement $(>20 \%)[10,26]$. In the current study, after the basic SIMT had been completed the average minimal clinicallyimportant improvement for functional exercise capacity and for the dyspnoea score only persisted for 6 months.

The present study contributes additional information to the knowledge regarding the benefits of IMT on exercise capacity and dyspnoea. It supports previous data that an outpatient program can provide benefits to patients with COPD.

A second important finding of the current study is that maintenance training should be applied. Otherwise, the useful benefit of the intensive basic training is not maintained beyond 6 months. Whether the maintenance training should be performed on a regular basis or by pulse training once in a while, is still to be determined.

The third important outcome of the current study, although comparison to an inpatient program was not made, is that IMT in patients with significant COPD may be performed at home, under some supervision, with relatively good compliance and with low monetary cost.

The current study was not designed to determine whether domiciliary inspiratory muscle training reduces exacerbations and hospital admissions in chronic obstructive pulmonary disease patients. Further studies should be performed in order to resolve these questions.

\section{References}

1. Gosselink R, Troosters T, Decramer M. Distribution of muscle weakness in patients with stable chronic obstructive pulmonary disease. J Cardiopulm Rehabil 2000; 20: 353-360.

2. Rochester DF, Braun NT. Determinants of maximal inspiratory pressure in chronic obstructive pulmonary disease. Am Rev Respir Dis 1985; 132: 42-47.

3. Pardy RL, Leith DE. Ventilatory muscle training. Respir Care 1984; 29: 278-284.

4. Smith K, Cook D, Guyatt GH, Madhavan J, Oxman AD. Respiratory muscle training in chronic airflow limitation: a meta-analysis. Am Rev Respir Dis 1992; 145: 533-539.

5. Weiner P, Berar-Yanay N, Davidovich A, Davidovich A,
Weiner M. The cumulative effect of long acting bronchodilators, exercise and inspiratory muscle training on the perception of dyspnea in patients with COPD. Chest 2000; 118: $672-78$

6. Hernandez MT, Rubio TM, Ruiz FO, Riera HS, Gil RS, Gomez JC. Results of a home-based training program for patients with COPD. Chest 2000; 118: 106-114.

7. Kim A, Larsen J, Covey M, Vitalo CA, Alex CG, Patel M. Inspiratory muscle training in patients with chronic obstructive pulmonary disease. Nurs Res 1993; 42: 356-362.

8. ACCP/AACVPR pulmonary rehabilitation guidelines panel. Pulmonary rehabilitation- Joint ACCP/AACVPR evidencebased guidelines. Chest 1997; 112: 1363-1395.

9. Lötters F, Van Tol B, Kwakkel G, Gosselink R. Effects of controlled inspiratory muscle training in patients with COPD: a meta-analysis. Eur Respir J 2002; 20: 570-576.

10. Troosters T, Gosselink R, Decramer M. Short- and longterm effects of outpatient rehabilitation in patients with chronic obstructive pulmonary disease: a randomized trial. Am J Med 2000; 109: 207-212.

11. Foglio K, Bianchi L, Bruletti G, Battista L, Pagani M, Ambrosino N. Long-term effectiveness of pulmonary rehabilitation in patients with chronic airway obstruction. Eur Respir J 1999; 13: 125-132.

12. American Thoracic Society. Standard for the diagnosis and care of patients with COPD. Am J Respir Crit Care Med 1995; 152: S78-S121.

13. McGavin CR, Gupta SP, McHardy GJR. Twelve-minute walking test for assessing disability in chronic bronchitis. Br Med J 1976; 1: 822-823.

14. Black LF, Hyatt RE. Maximal respiratory pressures: Normal values and the relationship to age and sex. $\mathrm{Am}$ Rev Respir Dis 1969; 99: 696-702.

15. Nickerson BG, Keens TG. Measuring ventilatory muscle endurance in humans as sustainable inspiratory pressure. J Appl Physiol 1982; 52: 768-772.

16. Martyn JB, Moreno RH, Pare PD, Pardy RL. Measurement of inspiratory muscle performance with incremental threshold loading. Am Rev Respir Dis 1987; 135: 919-923.

17. Mahler DA, Wells CK. Evaluation of clinical methods for rating dyspnea. Chest 1988; 93: 580-586.

18. Weiner P, Berar-Yanay N, Davidovich A, Magadle R, Weiner M. Specific inspiratory muscle training in patients with mild asthma with high consumption of inhaled $\beta_{2}$-agonists. Chest 2000; 117: 722-727.

19. el-Manshawi A, Killian KJ, Summers E, Jones NL. Breathlessness during exercise with and without resistive load. J Appl Physiol 1986; 61: 896-905.

20. Guell R, Casan P, Belda J, et al. Long-term effects of outpatient rehabilitation of COPD: a randomized trial. Chest 2000; 117: 976-983.

21. Ries AL, Kaplan RM, Limbeg TM, Prewitt LM. Effects of pulmonary rehabilitation on physiologic and psychosocial outcomes in patients with COPD. Ann Intern Med 1995; 122 : 823-832.

22. Strijbos JH, Potsma DS, Van Altena R, Gimeno F, Koeter GH. A comparison between outpatient hospital-based pulmonary rehabilitation program in patients with COPD. Chest 1996; 109: 366-372.

23. Pomerleau O, Adkins D, Pertschuk M. Predictors of outcome and recidivism in smoking cessation treatment. Addict Behav 1978; 3: 65-70.

24. Foreyt JP, Goodrick GK, Gotto AM. Limitations of behavioral treatment of obesity: review and analysis. J Behav Med 1981; 4: 159-174.

25. Martin JE, Dubbert PM. Exercise applications and promotion in behavioral medicine: current status and future directions. J Consult Clin Psychol 1982; 50: 1004-1017.

26. Cilione C, Lorenzi C, Dell Orso D, et al. Predictors of change in exercise capacity after comprehensive COPD inpatient rehabilitation. Med Sci Monit 2002; 8: CR7405. 